\title{
A Culling System for A Recipe using Sentiment Analysis Techniques
}

\author{
B.Surya Sai, P.Vamsi Mohan, Pijush Meher, M.Azhagiri
}

\begin{abstract}
This System helps the user for providing the recipes in which they are particularly interested in a mentioned criteria of ingredients on which the user wants to prepare a recipe. The input given by the user like the ingredient on which he/she wants to prepare a recipe can be of text, speech or visual. The process of providing a list of gathered recipes from different trusted and verified sources is performed with the help of Sentiment Analysis(SA), Watson Text-to-Speech and Watson Visual Recognition(WVR). The recipe extraction from different sources which are required for the user is retrieved with the help of a standard Web Crawler. The tools and technologies used for the proposed system are from Artificial Intelligence(AI), Natural Language Processing(NLP). The system proposed assists the user in providing a list of recipes in a prioritized order based on the optimization process performed by the Naïve Bayes Algorithm(NBA) of Sentiment Analysis. In addition, the displayed results of recipes have been reviewed and rated by different users from different sources.
\end{abstract}

Keywords : Nä̈ve Bayes Algorithm(NBA), Natural Language Processing $(N L P)$, Recipe extraction, Sentiment Analysis (SA), Web Crawler.

\section{INTRODUCTION}

If a person has to lead a fantastic life then he/she should be healthier and for being healthy they have to take the food which contains more vitamins and nutrients. At present, the world is erupting with a new technology dayby-day in everyday activities also. There is no time for selecting the food which contains more vitamins or nutrients instead people were eating the food which is there in front of them no matter what the food contains and run with the world like there is no other choice.

The best way to overcome this type of problem is to know what fruits or vegetables will be giving you the high nutritional values for leading a guaranteed life with the help of the technique proposed in our article. The main focus of the system is to provide the user with a recipe of his/her interest or preference.

This process works by taking the keywords given into account and performing feature selection, sentiment identification and classification of the sentiment. The process of optimization takes place after the above activities are performed. If the person gives an image as input then the name can be inferred with the help of visual recognition. If the input is given as audio then the speech is converted into text.

Revised Version Manuscript Received on Jun 20, 2019.

P. VamsiMohan, Department of ComputerScienceand Engineering, SRMIST, Chennai

Pijush Meher, Department of Computer Science and Engineering, SRMIST,Chennai,

M. Azhagiri, Department of Computer Science and Engineering, SRMIST, Chennai

B. Surya Sai, Department of Computer Science and Engineering, SRMIST, Chennai

The visual recognition and conversion of speech to text techniques are performed with the help of Watson services. Finally, the text which is the ingredient given or obtained(from speech and image) is taken and gets compared with the datasets which have already been provided then comes with an answer based on the categories they get divided.

The Sentiment Analysis technique can be classified as supervised, unsupervised, semi-supervised and reinforcement machine learning. The supervised machine learning is a process in which the input gets compared with the datasets which are already been defined and proposes a relationship between the input and output. Supervised machine learning mainly deals with classification and regression. Unsupervised machine learning is a technique used for producing a pattern detection and describing information in which the datasets involved have been trained to deduce the theory. Unsupervised machine learning mainly deals with clustering and association. Semi-supervised machine learning is a process where the description of labels may present or not. Reinforcement machine learning is a technique in which the experience of the environment and learns all the possible states to come up with a solution.

The system proposed assists the user with a set of on the criteria mentioned and helps them in choosing their interesting food with good nutritional values. For example, If the user mentions in the following the way as spicy Chow Mein then the process starts with searching of terms with spicy and Chow Mein separately from a given dataset and the input undergoes the technique of Sentiment Analysis then the optimization process and displays the set of results in a prioritized order. If the input is given in the form of visual then the snapshot undergoes comparison operation and comes up with a valid answer then follows the Sentiment Analysis.

\section{RELATED WORKS}

The Sentiment Analysis method used in [1] by KeijiYanai, Takuma Maruyama, and Yoshiyuki Kawano is Support Vector Machine(SVM) of linear kernel SVM. The system developed uses a visual recognition through which the snapshot of an ingredient present in a grocery store is given as input then performs an image recognition technology with the help of SURF feature.

M.B. Vivek, N. Manju and M.B. Vijay,[2]has developed a system for collaborative filtering approach provide recipe recommendation to the user based on the rating given on a recipe and profile preferences of the user. The approach taken based on the item is Tanimoto coefficient similarity and Log likelihood similarity for discovering the similarity between recipes. The approach based on user is Euclidian Distance and Pearson Correlation 
are used.The most accurate information is given by a userbased approach.

Opinion Mining is used in [3] by PakawanPugsee and MonsineeNiyomvanich, Non-members developed a system on polarity lexicon based through which the detection of polarity words, in turn, improves the analysis process from the comment analysis. The classification has been done in the form of positive, negative and neutral. This classification is based on the summation of the polarity of sentences in the comments.

The system developed in [4] by Rui Xia, Feng Xu, ChengqingZong, Qianmu Li, Yong Qi, and Tao Li , uses Dual Sentiment Analysis in which the Dual Training algorithm for the sentiment classifier and Dual Prediction algorithm for the classification of the reviews. The Dual Sentiment Analysis also makes uses of the 3-point (positive, negative and neutral) classification.

Sentiment Analysis technique is used for [5] by Kim Schouten and Flavius Frasincar provides information on how to distinguish the entities in sentiment mentioned within any aspects or documents of them. This mainly focuses on the entity or aspect level of analysis. The document which contains information can identify a sentiment in a piece of a word or a whole document which is considered whole as an entity.

Lakshmish Kaushik, Abhijeet Sangwan, [6] has developed the system to performs sentiment detection from audio with the help of Keyword Spotting(KWS) technique. For the selection of words from the speech, Maximum Entropy sentiment model is used.

The system developed in [7] by Sujata Rani and Prateek Kumar mainly focuses on the improvement of teaching and learning. The system uses the NRC emotion lexicon method in which the sentiment is divided into positive and negative and emotions into disgust, fear, anticipation, anger, joy, trust, sadness and surprise.

Sentiment Analysis used in [8] by Farkhund Iqbal, Jahanzeb Maqbool Hashmi, Benjamin C. M. Fung, (Senior Member, IEEE), Rabia Batool, Asad Masood Khattak, Saiqa Aleem , And Patrick C. K. Hung, developed a system uses a Genetic Algorithm which helps in reducing the feature set size without compromising the accuracy of the feature. For the evaluation of the developed model, the novel cross-disciplinary area of geopolitics as a case study.

\section{RECIPE SELECTION METHODOLOGY}

The Recipe Selection system uses the concepts of sentiment analysis and text summarization to give the rating for a recipe based on customers reviews by utilizing the input given from the user.

In the system that we proposed we take the user input as text, speech or images, and supportive words to get the rating. We use the web crawl application to get the reviews information and then we perform the summarization followed by sentiment identification and classification which comes under the sentiment analysis. Based on the results of the sentiment analysis we give the rating of the recipes to the user.

\section{A. Watson Speech-to-Text and Visual Recognition:}

The input if text can be the ingredients name such as potato, peas etc and any supportive words like healthy, spicy etc in our website. If the input is in the form of images or in the case where the user doesn't know about the ingredient he can use the photo to identify it in our site. We use the Watson visual recognition For that purpose, The Watson visual recognition of IBM which takes images as input and recognizes a name for the image. The recognition process happens in the form of image as the input. The classifiers involved are positive and negative. With the help of Watson Visual Recognition, one can build a custom build model. WVR can be incorporated into a custom made website through Bluemix. After the recognition of the image then converts into text.

The IBM Speech to text service provides APIs that use Speech recognition capabilities to produce a text of spoken audio by the human. This service can translate speech from various languages. When an input is given for the service then the detection of the keywords present in the speech given takes place and the conversion to text happens.

Steps:

1. Create an account in the IBM cloud.

2. Configure the services of Watson Visual Recognition and Speech to text.

3. Login into the account of Bluemix IBM with the credentials of IBM cloud.

4. Develop a required website.

5. Integrate the WVR and Speech-to-Text with the website using ID and password.

6. Describe the classifiers i.e., positive and negative for image and train the set of images.

7. Test an image and audio.

After this, the image or speech is taken into text format for our site.

\section{B. Web Crawl}

Web Crawl is additionally called as "Spider" which is fundamentally a program that peruses the internet and peruses the pages and other data in a determined and programmed way to make a few sections for web indexes like the Google, Bing and so on.

First, the web crawler begins with a rundown of URL's predefined to visit. At that point when visiting the URL's, it finds every one of the hyperlinks and data in the URL. The data is replicated and put something aside to reviewing and reading from live web. Thusly it slithers from one page to another and spreads over an enormous web partition with the goal that enough data is procured.

\section{Text Summarization}

Based on reviews obtained from web crawl is huge we prefer to use the text summarization technique to give faster and better output. Here we use the pronoun replacement based summarization. Before generating the summary a preprocessing is carried out which removes the noise, stop words such as a, the etc. in this process the pronouns which act as place holders for the nouns are replaced by the appropriate nouns and then extractive summarization is done. In the extractive text summarization, it looks for the most important keywords which deliver the opinion and the frequency of their occurrence. Then opinionated words are usually the adverbs, verbs and adjectives which are very well preserved by this method for further sentiment analysis. For a given text the Parts of Speech(POS) tagger tool is used to identify the

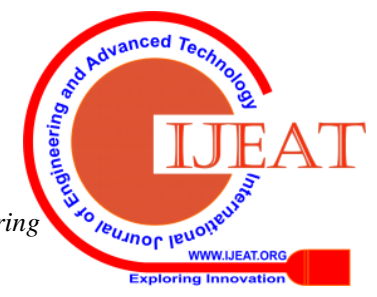


nouns, pronouns etc. the proper noun which is occurring close to the replaceable pronouns is used for replacement of that pronoun.

The next part is to eliminate stop words and computing the weight of keywords. Let's say Ws be the total number of stop words in the sentence, Wnbe the total number of keywords in it then the total sentence has,

$$
\mathrm{Wt}=\mathrm{Ws}+\mathrm{Wn}
$$

And Fm be the frequency of the $\mathrm{M}$ th keyword and $\mathrm{Wn}$ be the total number of keywords the weight of the $M$ th keyword is,

\section{Weightage of $\mathrm{M}$ th keyword $=\mathrm{Fm} / \mathrm{Wn}$}

Then the summation of the weightage of words in a sentence gives the sentence weightage. The sentence with higher weightage has higher priority to appear in the summary. The user determines the summary length by specifying the summary ratio $\mathrm{Sr}$ as,

Ns is the required length

$$
\mathrm{Ns}=(\mathrm{Sr} / 100) * \mathrm{Nt}
$$

$\mathrm{Nt}$ is the number of lines

$\mathrm{Sr}$ is the summary ratio

\section{Sentiment Analysis}

Sentiment analysis is used for extracting the subjective emotions and feelings from the given text.Naive Bayes is a prominent arrangement calculation for opinion mining. It is straightforward and can perform up to complex circumstances. For our characterization calculation, we are going to utilize the Naive Bayes classifier. The Naive Bayes classifier works by making sense of the likelihood of various qualities of information being identified with a specific class. The Naïve Bayes theorem is based on the Bayes theorem which is given as,

$\mathrm{P}(\mathrm{A} \mid \mathrm{B})=[\mathrm{P}(\mathrm{B} \mid \mathrm{A}) \cdot \mathrm{P}(\mathrm{A})] / \mathrm{P}(\mathrm{B})$

This basically states "the probability of A given that $\mathrm{B}$ is true equals the probability of $\mathrm{B}$ given that $\mathrm{A}$ is true times the probability of A being true, divided by the probability of B being true."

As the Bayes theorem doesn't work when there is one classification and two or more data point the naïve Bayes was introduced.

$\mathrm{P}(\mathrm{y} \mid \mathrm{x} 1, \ldots, \mathrm{xn})=\left[\mathrm{P}(\mathrm{y}) . \prod \mathrm{i}=1\right.$ to $\left.\mathrm{P}(\mathrm{xi} \mid \mathrm{y})\right] / \mathrm{P}(\mathrm{x} 1, \ldots, \mathrm{xn})$

This states that "the probability that classification $y$ is correct given the features $\mathrm{x} 1, \mathrm{x} 2$ and so on equals the probability of $y$ times the product of each $\mathrm{x}$ feature given $\mathrm{y}$, divided by the probability of the $\mathrm{x}$ features".

To find the right classification we just find out that in which classification $\mathrm{P}(\mathrm{y} \mid \mathrm{x} 1, \ldots, \mathrm{xn})$ has the highest probability.So we need to figure the probabilities of every order and the probabilities of each element falling into every arrangement for covering all potential outcomes. The easiest way to get the feature is to split up the sentence into words based on whitespaces. And use each word as a feature and count the number of times the occurrence of each word in negative reviews and in positive reviews and get word counts. This will help us in the long run to figure the probabilities of new audit having a place in each class.

As we now have the word counts we can convert them to probabilities and multiply them to get a predictable classification. Suppose we needed to ascertain the likelihood of the review "didn't like samosa" expresses a negative assessment. We locate the complete number of times "didn't" happen in the negative review and partition it by the absolute number of words in the negative reviews to get the likelihood of $\mathrm{x}$ given $\mathrm{y}$. We would do likewise for "like" and "samosa". At that point increase each of the three probabilities of a document expressing a negative opinion to get our last likelihood that the expresses negative sentiment.

We would do likewise for the positive sentiment and whichever probability is greater the review is assigned to that class(positive or negative). And if both probabilities are same would go to the neutral class. Now we can predict the reviews based on the probabilities.

In the system that we proposed we take the user input as text, speech or images and supportive words to get the rating. We use the web crawl application to get the reviews information and then we perform the summarization followed by sentiment identification and classification which comes under the sentiment analysis. Based on the results of the sentiment analysis we give the rating of the recipes to the user.

The development of the proposed system is mainly using Naïve Bayes algorithm for Sentiment Analysis, Pronoun Replacement algorithm for Text Summarization, Watson Visual Recognition for image identification and Speech to text conversion system in case of the speech input. The process starts with accepting input from the user of any form such as text, speech and image.If the input given is image then the execution starts with Watson Visual Recognition and gets converted to text. If the input given is speech then the process starts with the deduction of keywords from the speech and then converts into text. If the input is text or after the conversion of inputs (i.e., speech, image) to text then the process for retrieving the suitable recipe for the user starts.

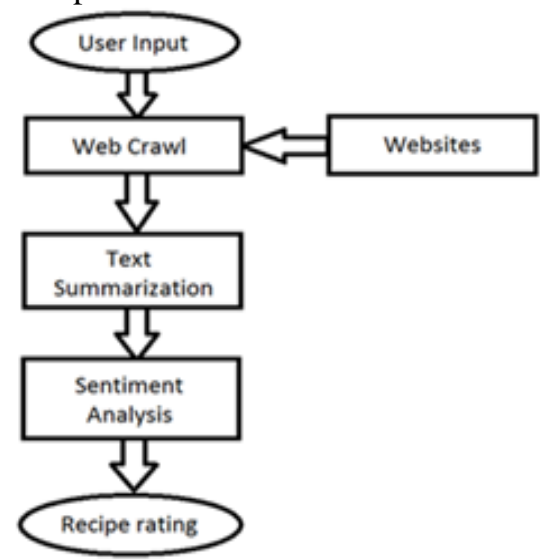

Figure-1: Proposed Architecture

Algorithm:

Input: Speech, Image, Text.

Output: Recipe rating in which the user is interested.

Procedure:

/*This program helps the user in the finding the recipe in which the user is interested*/

Begin

[1] The input of the form like speech, image and text is taken from the user onto the website.

[2] If the input is of the form image then

[A] Watson Visual Recognition takes the input of the image.

[B] Categorize the image based on the classifiers (i.e., positive and negative ) which have already been defined.

[C] Produces a valid name for the image which is a text. 
If(input==image)

\{ $/ *$ Image is taken as an input for Watson visual recognition and finds the name of the image*/

\}

[3] If the input is of the form speech then

[A] Watson Speech-to-Text takes the input of speech.

[B] Detect the keywords present in the speech.

[C] Forms a text and the text is forwarded.

If(input $==$ speech)

\{ $\quad / *$ Speech is taken as input for Watson speech-to-

Text and convert into text $* /$

\}

[4] The web crawl retrieves the page from the listed URL's and saves for the user based on the text.

queue=loadseed();

while(queue is not empty)

\{

URL gets dequeued

Document is requested

The document is stored for later processing

Links are obtained by parsing the document

Unseen links are added to the queue

\}

[5] The Pronoun Replacement algorithm filters the spaces, emoji's, etc., and summarizes the page based on the given input with Parts of Speech(POS) tool.

[6] The Naïve Bayes algorithm identifies the feelings and emotions present in the input then optimizes the summarized pages and displays them for the user.

I* where $\mathrm{x}_{1}, \mathrm{x}_{2}, \ldots, \mathrm{x}_{\mathrm{n}}=$ Features, $\mathrm{C}_{\mathrm{i}}=$ Class $* \backslash$

While(1)

\{

$\mathrm{P}\left(\mathrm{x}_{1}, \mathrm{x}_{2}, \ldots, \mathrm{x}_{\mathrm{n}}, \mathrm{C}_{1}\right)$

$\mathrm{P}\left(\mathrm{x}_{1} \mid \mathrm{x}_{2}, \ldots, \mathrm{x}_{\mathrm{n}}, \mathrm{C}_{\mathrm{i}}\right) . \mathrm{P}\left(\mathrm{x}_{2} \mid \mathrm{x}_{3}, \ldots ., \mathrm{x}_{\mathrm{n}}, \mathrm{C}_{\mathrm{i}}\right) \ldots . \mathrm{P}\left(\mathrm{x}_{\mathrm{n}-}\right.$

$\left.{ }_{1} \mid \mathrm{x}_{\mathrm{n}}, \mathrm{C}_{\mathrm{i}}\right) \cdot \mathrm{P}\left(\mathrm{x}_{\mathrm{n}} \mid \mathrm{C}_{\mathrm{i}}\right) \cdot \mathrm{P}\left(\mathrm{C}_{\mathrm{i}}\right)$

$\mathrm{i}++$;

\}

Select recipes above some probablity

End

Therefore, the user is provided with a list of recipes in which he/she is interested and go on for preparation of the recipe.

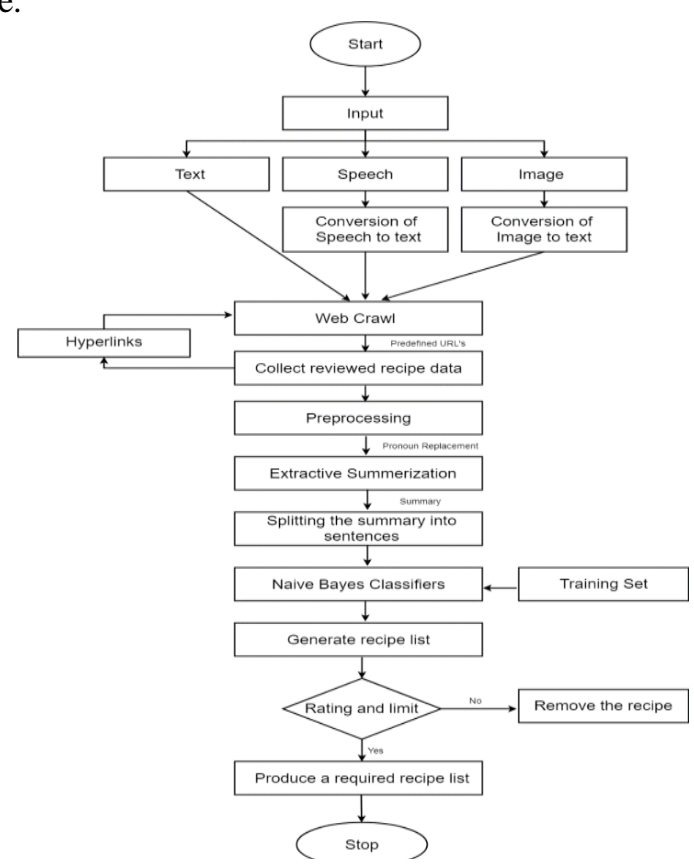

Figure-2:Flow diagram

\section{RESULTS AND DISCUSSION}

The system proposed is considered efficient based on the following parameters such as accuracy, performance, data integrity.With the help of the parameters the proposed system can be evaluated and efficiency of the system of the system can be found out. The accuracy of the system lies in detecting the name of the image got converted to text and conversion of speech to text. The performance of the system gives the time taken to produce a results. Data integrity helps the user in the trusting the system by providing a dataset in the background. This helps in the generating the output which is of more interest and accurate to the user.

\section{CONCLUSION}

The proposed system aims to reduce the searching efforts and time utilized by the user in searching a recipe to his liking. We use sentiment analysis and text summarization to reduce the search time and give the rating to the recipes. Our system supports text, audio and image as input where the user can give according to his needs. We use our Naïve Bayer's machine learning algorithm with text summarization to provide better results. The Naïve Bayer's gives the rating for the recipes based negative, positive and neutral words and finally provides an ordered list of recipes to the user based on the rating. As the text summarization helps for the Naïve Bayer's algorithm to provide better results than usually and the algorithm can be trained with different data sets and the efficiency can be measured.

\section{REFERENCES}

1. KeijiYanai, Takuma Maruyama and Yoshiyuki Kawano " A Cooking Recipe Recommendation System with Visual Recognition of Food Ingredients" International Journal of Interactive Mobile Technologies, Vol 8, No 2, 2014.

2. M.B. Vivek, N. Manju and M.B. Vijay "Machine Learning Based Food Recipe Recommendation System" D.S. Guru et al. (eds.), Proceedings of International Conference on Cognition and Recognition, Lecture Notes in Networks and Systems 14, Springer Nature Singapore Pte Ltd. 2018.

3. PakawanPugsee and MonsineeNiyomvanich "Sentiment Analysis of Food Recipe Comments"ECTI Transactions on Computer And Information Technology Vol.9, No.2 November 2015.

4. Rui Xia, Feng Xu, ChengqingZong, Qianmu Li, Yong Qi, and Tao Li "Dual Sentiment Analysis: Considering Two Sides of One Review" IEEE Transactions on Knowledge And Data Engineering, Vol. 27, No. 8, August 2015.

5. Kim Schouten and Flavius Frasincar "Survey on Aspect-Level Sentiment Analysis" IEEE Transactions on Knowledge AndData Engineering, Vol. 28, No. 3, March 2016.

6. Lakshmish Kaushik, Abhijeet Sangwan and John H. L. Hansen "Automatic Sentiment Detection in Naturalistic Audio" IEEE/ACM Transactions on Audio,Speech, And Language Processing, Vol. 25, No. 8, August 2017.

7. Sujata Rani and Parteek Kumar "A Sentiment Analysis System to Improve Teaching and Learning" IEEE Computer Society, Vol 50,No.5 May 2017.

8. Farkhund Iqbal, Jahanzeb Maqbool Hashmi, Benjamin C. M. Fung Rabia Batool, Asad Masood Khattak, Saiqa Aleem, And Patrick C. K. Hung "A HybridFramework for Sentiment Analysis Using Genetic Algorithm Based Feature Reduction" IEEE Access, Vol 7,2019 . 


\section{AUTHORS PROFILE}

P. VamsiMohan, Department of ComputerScienceand Engineering, SRMIST, Chennai

Pijush Meher, Department of Computer Science and Engineering, SRMIST,Chennai,

M. Azhagiri, Department of Computer Science and Engineering, SRMIST, Chennai

B. Surya Sai, Department of Computer Science and Engineering, SRMIST, Chennai 